\title{
DESPRENDIMIENTO PREMATURO DE PLACENTA NORMOINCERTA
}

A PROPOSITO DE 66 CASOS EN EL HOSPITAL SAN JUAN DE DIOS, DE CUCUTA.

Dr. Alberto Duarte-Contreras*

Dr. Manuel José Palau C.**

Al revisar la mortalidad perinatal de nuestro Servicio Obstétrico, encontramos que el $17,69 \%$ nos la proporciona el Desprendimiento Prematuro de Placenta Normoincerta (D. P. P. N.) (1) (2). También hemos podido comprobar que el DPPN ocupa entre nosotros el segundo lugar como causante de hemorragia en el tercer trimestre del embarazo. $Y$ si a esto agregamos la incertidumbre que aún persiste hoy en día sobre sus factores etiopatogénicos, mas el reducido número de estudios publicados al respecto en nuestro país, nos preocupamos por este "síndrome clínico" y vemos la necesidad de hacer una revisión total de nuestra estadística con miras a discutir el tratamiento que hemos seguido, y a estudiar las pautas y el manejo que en el futuro ofrezca una mayor seguridad tanto al obstetra como a las pacientes.

\section{II - Material y Métodos}

Hemos revisado las Historias clínicas de 14.161 partos atendidos en el Hospital San Juan de Dios, de Cúcuta, Colombia, en el lapso comprendido entre el 1\% de Sejtiembre de 1960 y el 31 de Diciembre de 1968.

El diagnóstico de DPPN lo hemos hecho mediante el examen y hallazgos clínico obstétricos; el estudio histopatológico de placenta lo practicamos solamente en uno de los casos.

* Médico Jefe del Departamento de Obstetricia y Ginecología del Hospital San Juan de Dios, de Cúcuta, Colombia.

** Médico Residente (R4) del Departamento de Obstetricia y Ginecología del Hospital - San Juan de Dios, de Cúcuta.

\section{III - Frecuencia CUADRO 1 \\ DPPN - FRECUENCIA}

\begin{tabular}{lcccr}
\hline Autor & $N^{0}$ de Partos & $N^{0}$ DPPN & $\%$ & Uno por cada \\
\hline Arcay (3) & 17.675 & 340 & 1,90 & 52 \\
Decker (4) & 25.030 & 312 & 1,24 & 80 \\
Dyer (5) & 11.556 & 214 & 0,54 & 180 \\
Rouchy (6) & 27.447 & 150 & 0,53 & 183 \\
Ramírez (7) & 21.946 & 79 & 0,35 & 278 \\
Ochoa (8) & 39.795 & 132 & 0,33 & 301 \\
Londoño (9) & 8.495 & 27 & 0,32 & 315 \\
Rozo (10) & 44.119 & 139 & 0,31 & 317 \\
Salinas (11) & 12.105 & 28 & 0,23 & 432 \\
Pundel (12) & 26.487 & 50 & 0,18 & 529 \\
Ortiz (13) & 28.232 & 43 & 0,17 & 588 \\
Karchmer (14) & 83.294 & 125 & 0,15 & 666 \\
Presente estudio & 14.169 & 66 & 0,46 & 214 \\
\hline
\end{tabular}


Al comparar los diferentes trabajos publicados tanto en la literatura nacional como extranjera observamos que la frecuencia del DPPN varía mucho yendo desde 1 por cada 52 partos (3) hasta 1 por cada 666 (14), y aún citan hasta 1 por cada 1.500 (24). Nuestra incidencia de 1 por cada 214 partos $(0,46 \%)$ está próxima a la encontrada por los autores colombianos Rozo (10), Londoño (9) y Ochoa (8). Podemos observar con toda claridad al dilucidar las cifras dadas en los diferentes trabajos, que este porcentaje de incidencia disminuye entre más alto sea el nivel de los grupos estudiados y a medida que haya una mejor motivación de la consulta prenatal.

\section{III - Edad de las pacientes \\ CUADRO 2 \\ DPPN - EDAD DE LAS PACIENTES}

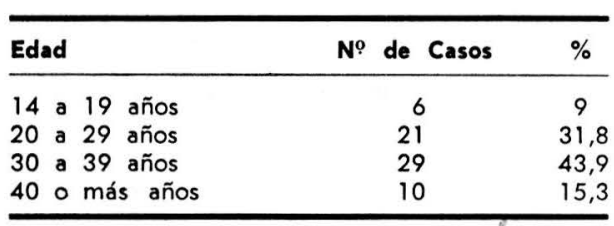

En nuestra serie la paciente de menor edad fue una primigesta de 14 años y la de mayor edad una multigesta ( $G$ 12) de 43 años. Aun cuando encontramos la mayor frecuencia en pacientes con edad superiro a los 30 años $(59,2 \%)$, edad propia de las grandes multíparas, no podemos deducir por ello que en nuestro medio haya relación alguna entre la edad de las pacientes estudiadas y la aparición de esta complicación obstétrica.

\section{IV - Paridad}

Encontramos diez pacientes primigestas $(15,3 \%)$ y $56(84,7 \%)$ multigestas, y de estas últimas, 30 eran grandes multíparas $(45,4 \%)$ con sie-
Rev. Col. Obst. y Ginec.

\section{CUADRO 3}

DPPN - PARIDAD

\begin{tabular}{|c|c|c|c|}
\hline Paridad & $N^{o} d$ & Casos & $\%$ \\
\hline G I & & 0 & 15,3 \\
\hline$G \quad I I$ a $V \mid$ & & 6 & 39,3 \\
\hline G VII ○ más & & 0 & 45,4 \\
\hline
\end{tabular}

te partos o más; ello nos confirma la apreciaicón que hicimos al estudiar la edad de las pacientes de que la mayor incidencia del DPPN se presenta en mujeres con edad superior a los 30 años, edad que corresponde a las grandes multíparas.

Debemos observar que no encontramos embarazos gemelares en los casos estudiados, mas sí una recidiva.

\section{V - Edad del embarazo}

\section{CUADRO 4}

$$
\text { DPPN - EDAD DEL EMBARAZO }
$$

\begin{tabular}{|c|c|c|c|}
\hline $\begin{array}{l}\text { Semanas de } \\
\text { embarazo }\end{array}$ & No & de Casos & $\%$ \\
\hline 28 a 35 & & 29 & 46,1 \\
\hline 36 ○ más & & 37 & 53,9 \\
\hline
\end{tabular}

No tuvimos en cuenta para el presente estudio el DPPN en embarazos menores de 28 semanas, pues los consideramos como abortos.

La mayor frecuencia la encontramos en embarazos de 36 semanas o más ( 37 casos, 53,9\%), sin que nos parezca esta cifra significativamente superior a la observada en embarazos de 28 a 35 semanas (20 casos, $46,1 \%)$ ).

Investigamo scon mucha acucia la observación de Bierber (15), comprobada por Ochoa (8), de que el DPP $\mathrm{N}$ ocurre con mayor facilidad en las semanas $28,32,36 \circ 40$, sin que lo hubiésemos podido constatar. 
VI - Control prenatal

CUADRO 5

DPPN - CONTROL PRENATAL

\begin{tabular}{|c|c|c|}
\hline & No de Casos & $\%$ \\
\hline Con control & 17 & 25,7 \\
\hline Sin control & 49 & 74,3 \\
\hline
\end{tabular}

Solamente 17 pacientes habían tenido control prenatal; las demás, 49 pacientes $(74,3 \%)$ buscaron nuestro Servicio Obstétrico cuando los síntomas propios de esta contingencia les hacían temer por su vida o por la de su futuro hijo.

Como veremos luego, cuatro de las pacientes que llegaron al Servicio después de cuatro horas de haber iniciado hemorragia genital, habían asistido a la consulta prenatal; puede ello indicarnos que adolece nuestra consulta prenatal de una mejor motivación y de una educación más práctica para quienes a ella asisten, a más de que nos habla muy a las claras del bajo nivel cultural del grupo estudiado.

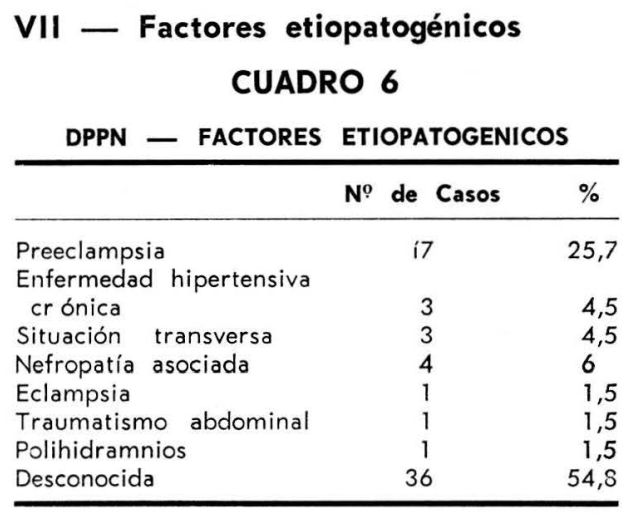

La toxemia dio un alto tributo como posible factor causal habiéndola encontrado en 18 casos en total, de los cuales $17(25,7 \%)$ fueron preeclampsias y uno $(1,5 \%)$ eclampsia.
La nefropatía asociada la hallamos en 4 casos $(6 \%)$, y en uno de ellos muy severa. La enfermedad hipertensiva crónica se hizo presente en 3 casos $(4,5 \%)$. El traumatismo abdominal por golpes directos recibidos lo observamos solamente en un caso $(1,5 \%)$. La sobredistensión uterilna por polihidramnios la vimos en una paceinte $(1,5 \%)$, y la situación transversa en tres. $Y$ en 36 no encontramos agente causal alguno al que pudiésemos atribuir la aparición de tan temida complicación obstétrica.

Estos factores ya enunciados serían en realidad los causantes del desencadenamiento del DPPN? Imposible responder de manera afirmativa a excepción del caso en el cual un traumatismo local cierto hizo iniciar el desprendimiento. Los demás factores encontrados los consideramos como simples coadyuvantes al tratar de dilucidar el mecanismo etiopatogénico del abruptio en el grupo de pacientes que estudiamos.

La teoría de la escuela uruguaya relaciona la aparición de este síndrome a un vasoespasmo arterial que produce un alza del tono uterino, formando y aumentando el hematoma en círculo vicioso (21); teoría no aceptada por muchos autores.

Ghosn sostiene (19) que así como para la aparición o desencadenamiento de toda hemorragia súbita y espontánea se requiere una sacudida tensional que actúe sobre un terreno frágil, de la misma manera cualquier exitación agresiva que actúe sobre un terreno hipertensivo crónico anterior al embarazo, o sobre un fondo de toxemia gravídica, o aún sobre un sistema vascular aparentemente normal, ejercería su acción sobre una caduca frágil y de vasos muy vulnerables. 
Según los casos, la causa inicial es el terreno general (hipertensión, toxemia), o la exitación violenta (traumatismo moral, gran emoción, disfunción neuro hormonal gravídica), - la extrema fragilidad de la caduca y de su sistema vascular.

\section{VIII - Evolución}

\section{CUADRO 7}

\begin{tabular}{lcc}
\multicolumn{4}{c}{ DPPN } & No de Casos & $\%$ \\
\hline \multicolumn{4}{c}{ EVOUCION } \\
\hline Menos de 4 horas & 27 & 41 \\
4 a 8 horas & 10 & 15,2 \\
8 a 12 horas & 3 & 4,5 \\
12 a 24 horas & 2 & 3 \\
más de 24 horas & 2 & 3 \\
ignorada & 22 & 33,3 \\
\hline
\end{tabular}

Unicamente 27pacientes (41\%) ingresaron al Servicio antes de 4 horas de haber iniciado la sintomatología. Diecisiete pacientes $(25,7 \%)$ llegaron después de 4 horas de presentar los síntomas propios, y dos de éstas (3\%) esperaron más de 24 horas para su hospitalización. En 22 casos $(33,3 \%)$ desconocemos el tiempo de evolución.

Es de lógica que la demora en la hospitalización aunada a la falta de control prenatal, aumenta los riesgos tanto maternos como fetales.

Dieciseis de las pacientes que ingresarno con una evolución menor de cuatro horas, presentaban copiosa hemorragia y tres de ellas se encontraban en estado de shock, con feto muerto y desprendimiento total de placenta.

Cabe anotar que el pronóstico materno fetal no está de un todo en relación directa con el tiempo de evolución del desprendimiento, sino con la intensidad de la hemorragai y con la superficie de placenta desprendida.

\section{IX - Cuadro clínico}

CUADRO 8

DPPN - CUADRO CLINICO

\begin{tabular}{lcc}
\hline Síntomas & No $^{\mathbf{c}}$ de Casos & $\%$ \\
\hline Dolor & 24 & 36,4 \\
Hipertonía & 18 & 26,4 \\
Sangrado moderado & 37 & 56,2 \\
Sangrado abundante & 29 & 43,8 \\
Shock & 8 & 12 \\
Sufrimiento fetal & 11 & 16,6 \\
MUERTE FETAL & 28 & 42,4 \\
\hline
\end{tabular}

En el cuadro clínico que presentaron nuestras pacientes cuando ingresaron al Servicio predominó el dolor ( 24 casos, 36,4\%) sobre la hipertonía ( 18 casos, 26,4\%). Estos dos síntomas más el sangrado genital que lo presentaron todas, forman la tríada sintomática que facilita el diagnóstico de este alarmante síndrome.

Ocho pacientes (12\%) ingresaron en estado de shock: cuatro con sangrado abundante (6\%) y cuatro con sangrado excesivo.

A 24 pacientes $(36,4 \%)$ les suministramos transfusiones de sangre total en cantidad que fue de los 500 a los 2.000 cc.

En 19 pacientes $(28,7 \%)$ se presentó el DPPN sin que hubieran iniciado el trabajo de parto: la sintomatología en ellas fue muy dramática, habiendo muerto una por shock hipovolémico irreversible.

En 11 pacientes $(16,6 \%)$ diagnosticamos sufrimiento fetal; y tres de estos fetos murieron in útero después de ingresar las pacientes al Servicio.

En 28 pacientes $(42,4 \%)$ hicimos y comprobamos el diagnóstico de muerte fetal en el examen de ingreso. 
X - Complicaciones

CUADRO 9

DPPN - COMPLICACIONES

\begin{tabular}{lcc}
\hline & $N^{0}$ de Casos & $\%$ \\
\hline Atonía uterina & 5 & 7,6 \\
lleus paralético & 3 & 4,5 \\
Sepsis puerperal & 3 & 4,5 \\
Insuficiencia renal & 2 & 3 \\
Ruptura uterina & 2 & 3 \\
Hipofibrinogenemia & 2 & 3 \\
Sin complicaciones & 49 & 74,2 \\
\hline
\end{tabular}

Cuarenta y nueve de nuestras pacientes $(74,2 \%)$ evolucionaron normalmente; $17(24,6 \%)$ presentaron diferentes complciaciones y en ellas el pronóstico fué incierto y sombrío con un tributo de dos pacientes a la mortalidad.

La atonía uterina la observamos en 5 pacientes $(7,5 \%)$; el íleo paralítico en $3(4,5 \%)$; la sepsis puerperal en 3 ; la insuficiencia renal en 2 $(3 \%)$. Dos rupturas uterinas espontáneas fueron ocasionadas sin duda alguna por la administración mal controlada en ocitócicos. Nos llama la atención la baja incidencia de hipofibrinogenemia, complicación de elevado porcentaje en varias de las estadísticas revisadas, en las cuales alcanza un 16 (14) y hasta un $23,4 \%$ (8).

\section{XI - Muerte materna}

Tuvimos dos muertes maternas (3\%): una por hipofibrinogenemia y otra por shock hipovolémico; mortalidad ésta que no podemos considerar elevada si la comparamos con los datos suministrados por diferentes autores y que consignamos en el Cuadro 10.
CUADRO 10

DPPN - MUERTE MATERNA

\begin{tabular}{llll}
\hline Autor & \multicolumn{2}{l}{ Incidencia } & $\%$ \\
\hline Perlin (16) & 1 entre 197 & 0,50 \\
Studdifort (17) & 2 & 305 & 0,66 \\
Ochoa (8) & 1 & 132 & 0,75 \\
Daro (18) & 6 & 301 & 1,90 \\
Ghosn (19) & 2 & 72 & 2,6 \\
Dyer (5) & 6 & 214 & 2,8 \\
Arcay (3) & 2 & 52 & 3,84 \\
Karchmer (14) & 6 & 125 & 4,8 \\
Rozo (10) & 9 & 139 & 6,47 \\
Suárez (20) & & & 7,35 \\
Pundel (12) & 5 & 50 & 10 \\
Presente estudio & 2 & 66 & 3 \\
\hline
\end{tabular}

XII - Mortalidad perinatal

CUADRO 11

DPPN - MORTALIDAD PERINATAL

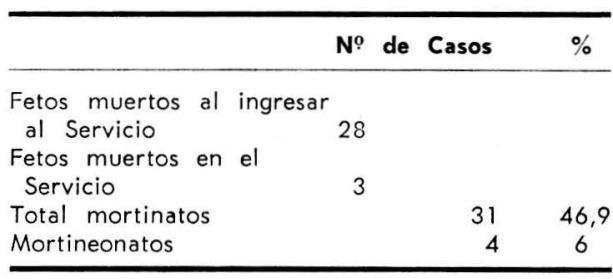

De los 66 productos obtenidos, 35 nacieron vivos, pero 4 de ellos ( $6 \%$ ) murieron durante las seis primeras horas. Solamente sobrevivieron y salieron del Servicio en buenas condiciones 31 niños $(47,1 \%)$.

Hubo 31 mortinatos (46,9\%): 28 óbitos $(42,4 \%)$ al ingresar la Servicio y $3(4,5 \%)$ que ingresaron con gran sufrimiento fetal y murieron en la Maternidad antes de la atención del parto.

En esta forma nuestra mortalidad perinatal no corregida fue de $35 \mathrm{ca}$ sos $(52,9 \%)$, y la corregida fue de 7 casos $(10,6 \%)$, cifras estas que bien podemos comparar con las encontradas por otros autores. 
CUADRO 12

DPPN - MORTALIDAD PERINATAL

\begin{tabular}{lcc}
\hline Autor & No corregida \% & Corregida $\%$ \\
\hline Hendelman (21) & 31 & \\
Perlin (16) & 40 & \\
Bierber (15) & 40,3 & \\
Ochoa (8) & 59,1 & \\
Karchmer (15) & 68 & 7.2 \\
Hester (22) & 68 & \\
Rozo (10) & 68,18 & \\
Salinas (11) & 70,8 & \\
Ghosn (10) & 75 & \\
Arcay (13) & 17 & \\
Londoño (9) & 100 & \\
Pres@nte estudio & 52,9 & 10,6 \\
\hline
\end{tabular}

El diagnóstico de muerte fetal lo hicimos ante la ausencia de ruidos fetales, pero admitimos que en presencia de una contractura uterina puede hacerse imposible la auscultación. En estos casos deberíamos recurrir a la electrofonocardiografía o al uso del estetoscopio electrónico para hacer posible un diagnóstico seguro: nosotros carecemos de estos auxiliares clínicos.
El pronóstico de supervivencia fetal se ensombrece en relación directa al tiempo que transcurra entre la iniciación del clesprendimiento y la hora en que se efectúe el parto, lo mismo que en relación directa a la cantidad de hemorragia que se presente, a la edad del embarazo, y sobre todo a la superficie de placenta desprendida.

Así, en nuestro estudio, en 28 pacientes $(42,4 \%)$ que presentaron un desprendimiento total o casi total de placenta, la mortalidad fetal fue del $100 \%$; en 11 casos $(16,6 \%)$ en los cuales el desprendimiento de placenta alcanzó de un 20 a un $50 \%$, encontramos tres mortinatos y cuatro mortineonatos, y una supervivencia de apenas cuatro productos. $Y$ en 27 pacientes $(41 \%)$ que presentaron un desprendimiento cercano pero inferior al $20 \%$ de la superficie placentaria, la supervivencia de los productos fue del $100 \%$, si bien 21 de ellos tuvieron un Test de Apgar entre $4 / 10$ y $6 / 10$.

XII - Peso de los productos

CUADRO 12 DPPN - PESO DE LOS PRODUCTOS

\begin{tabular}{lcccc}
\hline Peso en gramos & No Casos & $\%$ & Mortalidad perinatal & Sobrevivieron No. \\
\hline 1.000 a 1.500 & 12 & 18,8 & 11 & 1 \\
1.501 a 2.000 & 12 & 18,8 & 9 & 3 \\
2.001 a 2.500 & 14 & 21,2 & 4 & 10 \\
2.501 a 3.000 & 11 & 16,6 & 2 & 9 \\
3.001 a 3.500 & 6 & 8 & 2 & 4 \\
3.501 o más & 4 & 6 & 0 & 4 \\
desconocido & 7 & 10,6 & 7 & 0 \\
\hline
\end{tabular}

No apreciamos en nuestra casuística cifras que puedan tener significación alguna especial, ya que la relación encontrada entre peso de los productos y mortalidad perinatal es la expresada comunmente cuando se estudian síndromes hemorrágicos del tercer trimestre del embarazo. En cambio es muy significativo el índice de prematuridad ( 38 casos en total, $58,8 \%$ ) como también el alto tributo que ella rindió a la mortalidad perinatal, ya que el $68,5 \%$ de la mortalidad perinatal se hizo a expensas de los productos de bajo peso. 


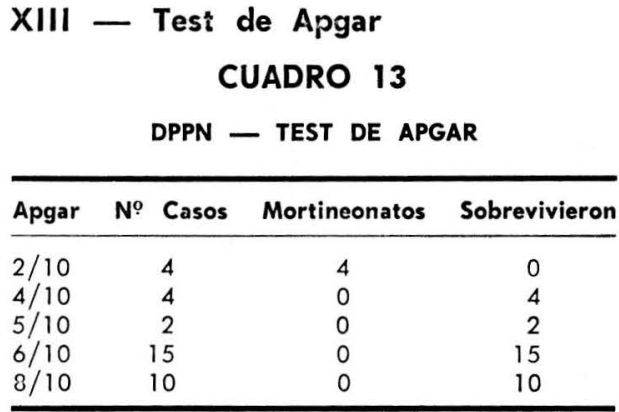

Todos los productos con un test de Apgar inferior a 4/10 fallecieron en las primeras 6 horas de vida. Quienes nacieron con Apgar 4/10 o superior a él, sobrevivieron. Observamos que los cuatro mortineonatos están incluídos enrte los productos de bajo peso.

\section{XIV - Manejo de las pacientes CUADRO 14 \\ DPPN - MANEJO DE LAS PACIENTES}

\begin{tabular}{|c|c|c|c|}
\hline Atención obstétrica & No de & Casos & $\%$ \\
\hline Parto vaginal espontáneo & 3 & & 50,0 \\
\hline Parto vaginal con Vacuum & & 5 & 7,5 \\
\hline Parto vaginal con fórceps & & 2 & 3 \\
\hline extracción podálica & & 1 & 1,5 \\
\hline Parto por cesárea Kerr & 2 & & 36,5 \\
\hline Parto por cesárea corporal & & 1 & 1,5 \\
\hline
\end{tabular}

Sin duda alguna el manejo de estas pacientes requiere una perfecta orientación y una constante y escrutadora vigilancia de parte del obstetra, dado el alto riesgo tanto materno como fetal que implica esta desafortunada contingencia.

La conducta que se deba seguir en estas pacientes depende de múltiples y muy diversos factores: edad del embarazo, cuadro clínico, presencia o ausencia de trabajo de parto, estado del cuello, estado de la madre y del feto, complicaciones entre las cuales debemos prestar especial cuidado a la hipofibrinogenemia.

Cuarenta y una de nuestras pacientes $(62 \%)$ tuvieron parto vaginal; y a 25 les practicamos operación cesárea $(38 \%)$. O sea: el parto vaginal prevaleció en nuestra revisión en una relación aproximad ade 2 a 1,2.

En lo que atañe a operación cesárea, ésta la realizamos en tres contingencias: a tres pacientes $(4,5 \%)$ a quienes la copiosa hemorragia había puesto en serio peligro su vida; a 19 pacientes $(29 \%)$ en quienes la evolución del trabajo (borramiento, dilatación, encajamiento) hicieron prever que el parto no se realizaría en corto tiempo, amenazando así la supervivencia del producto quien ya presentaba síntomas claros de sufrimiento fetal; y a tres pacientes por diferentes indicaciones obstétricas pertinentes a cesárea (39).

Empleamos el Vacuum Extractor para acelerar y conducir el descenso; y el fórceps lo usamos únicamente en el desprendimiento.

Si valorada la paciente tomamos la decisión de atender el parto por vía vaginal, practicamos la amniorrexis de inmediato siempre y cuando nos lo permitan las condiciones cervicales. La amniotomía puede producir una rápida aceleración de la dilatación cervical (25) (26) (27) (28) (29); y cuando a esta maniobra agregamos la administración de ocitocina a la dosis de 2 a 8 miliunidades por minuto, obtenemos un aumento de la intensidad y en la frecuencia de las contracciones a más de su coordinación, no obstante la existencia de gran actividad e hipertonía uterina (30) (31) (32) (33).

Con alguna frecuencia usamos la Meperidina (Demerol) a la dosis de 100 miligramos por vía muscular, 
que si bien carece de efecto sobre la contractilidad uterina en el abruptio, sí calma el dolor continuo producido por la hipertonía (26) (27).

Si practicada la amniotomía más la aplicación de ocitocina, no se acelera la dilatación cervical, podemos emplear el Vacuum Extractor en multíparas, y en esta forma, haciendo una tracción sostenida y suave, obtendremos una rápida dilatación cervical (29), maniobra ésta que como la amniotomía puede producir por vía refleja bien sea un incremento en la actividad contráctil del útero mejorando y acelerando el trabajo, o un aumento del flujo sanguíneo renal por el mecanismo reflejo útero renal de Trueta, evitando así en grna parte las complicaciones renales (14).

Revisamos las placentas y observamos casi en todas ellas zonas de infartos de muy diversa localización, aspecto y tamaño; pero únicamente una placenta fue sometida a examen anatomopatológico (falla protuberante del Servicio) habiéndose encontrado en ella dilatación de la vena umbilical.

El suministro de expansores de plasma o de transfusiones de sangre total debe ser oportuno y suficiente.

Motivo de gran atención para nosotros es el puerperio: tratamos el estado anémico consecutivo por diferentes métodos a más de las complicaciones y de la patología sobreagregada. Hemos observado que la hemorragia post parto es una complicación muy común en estas pacientes, y por ello administramos ocitócicos de manera rutinaria, mas no así la antibiotictoerapia, la que empleamos solamente cuando la creemos oportuna o necesaria.

La hipofibrinogenemia la encontramos en dos casos. Conocedores de los diversos mecanismos de producción (coagulación intravascular difusa o desfibrinación; aumento de la actividad fibrinolítica; o por depósitos de fibrina en el hematoma retroplacentario) (34) (35) (36) (37) (38), instituímos el tratamiento en una paciente con áciclo epsilén amino caproico y transfusión con excelente resultado; y en la otra usamos fibrinógeno, hidrocortisona y transfusión: esta paciente flaleció.

\section{XV - Resumen}

Al estudiar 14.161 historias de partos atendidos en el Hospital San Juan de Dios de Cúcuta, Colombia, encontramos 66 pacientes con D.P.P.N. $(0,46 \%)$, y de ellas solamente el $25,7 \%$ habían recibido control prenatal.

No observamos relación entre la edad de las pacientes y el DPPN, mas no así con la paridad, ya que el $84,7 \%$ se presentó en multigestas. Tampoco observamos relación con la edad del embarazo, si bien en el $58,8 \%$ obtuvimos productos de bajo peso.

Los factores etiopatogénicos son muy inciertos en nuestra casuística.

La evolución varió desde una vez iniciada la hemorragia hasta más de 24 horas después; y como síntoma predominó el dolor sobre la hipertonía.

El $62 \%$ de las pacientes tuvieron parto vaginal y se practicó cesárea al $38 \%$. La hipofibrinogenemia sólo la pudimos comprobar en dos casos.

La mortalidad perinatal corregida fue de $10,6 \%$. Se presentaron dos muertes maternas.

Por último se analiza el manejo de las pacientes. 


\section{BIBLIOGRAFIA}

1 DUARTE CONTRERAS ALBERTO, CORONEL G. Y BUSTAMANTE GILBERTO: Mortalidad perinatal en el Hospital San Juan de Dios de Cúcuta. A propósito de 3.365 historias. Rev. Col. Cbst. y Gin. 14; 413-423, 1963.

2 DUARTE CONTRERAS ALBERTO, CORONEL. JOSE G. Y BUSTAMANTE GILBERTO: Mortalidad perinatal en el Hospital San Juan de Dios de Cúcuta. Estudio de 4.323 historias. Rev. Col. Obst. y Gin. 15; 319-331, 1964.

3 ARCAY A.: Desprendimiento prematuro normoplacentario. Rev. Obst. Gin. Venez. $17 ; 723,1957$.

5 DYER ISIDORE and MCCAUGHEY EVERETT $\checkmark$.: Abruptio placentae. Ten year survey. Am. J. Obst. Gyn. 77; 1176, 1959.

6 ROUCHY J., CREZE, R.: LE DALL et GROSSIEUX P.: Diagnostic et traitement de I'hémorragie rétro-placentaire (étude de 150 observations). Gyn. et Obst. 64; 37 , 1965.

7 RAMIREZ SOTO EZEQUIEL Y KARCHMER SAMUEL. Desprendimiento prematuro de placenta normoincerta. Análisis de 79 casos. Mem. de la I Jornada Médica Bienal del H. G. O. No 1 del I. M. S. S. Tomo l; 285,1964

8 OCHOA M. GERMAN y RESTREPO A. Luis FERNANDO. Abruptio placentae. Análisis clínico de 132 casos. Rev. Col. Obst. Gin. 15 ; 369, 1964

9 LONDOÑO A. Hematoma retroplacentario. Tesis de grado. U. de Antioquia. 1945.

10 ROZO L. F. Desprendimiento prematuro de la placenta normoincerta en el Instituto Materno Infantil Concepción Villaveces de Acosta. Rev. Col. Obst. Gin. 12; 162, 1961.

11 SALINAS FALERO JUAN ARTURO. Desprendimiento prematuro de placenta normoincerta. Análisis de 28 casos y valoración de su terapéutica. Gin. Obst. Méx. 18; $257,1963$.

12 PUNDEL J. P. et REILES $M$. Le décollement prématuré du placenta normalement inse. ré. Gyn. et Obst. 60; 85, 1962.

13 ORTIZ ARROYO ROSENDO. Análisis clínico de 48 casos de desprendimiento prematuro de placenta normoincerta. Mem. de la I Jornada Méd. Bienal del H. G. O. No 1 del I.M.S.S. Tomo I; 277, 1964
14 KARCHMER SAMUEL, RUIZ CEBALLOS ROBERTO, PEREZ DIAZ ANTONIO Y PAVON MONCADA MARIO. Desprendimiento prematuro de placenta normoincerta. Análisis de 125 casos. Gin. Obst. Méx. 24; 135, 1968.

15 BIERBER G. F. Review of three hundred fifthy three cases of premature separation of the placenta. Am. J. Obst. Gyn. 65; $257,1953$.

16 PERLIN I. A., and STEWART JOHN. Abruptio placentae. A Clinical review of $197 \mathrm{ca}$ ses. Am. J. Obst. Gyn. 85; 284, 1963.

17 STUDDIFORD WILLIAM E. and DECKER WAYNE $H$. Management of premature separation of placenta. Obst. and Gyn. 1; 418, 1953

18 DARO A. F. Premature separation of the normally implanted placenta. Am. J. Obst. Gyn. 72; 599, 1953.

19 GHOSN G. et KHALIFE M. Apoplexie utéro placentaire. A propos de 72 cas en six ans a la Maternité francaise de Beyrouth. Gyn. et Obst. $67 ; 213,1968$.

20 SUAREZ HERRERA R. Rev. Obst. Gin. Venez. Memorias del Primer Congreso Ven 3zolano de Obst. Gin. 168, 1955.

21 HENDELMAN $M$. and FRASSER W. D. A. Clinical Analysis of abruptio placentae. Am. J. Obst. Gyn. 80; 17, 1960.

22 HeSTER L. L. and SALLEy J. Am. J. Obst. Gyn. 1218, 1957

23 WEINER ALBERT E., REID DUNCAN E. and ROBY CHARLES C. Incoagulable blood in severe premature separation of placenta; method of management. Am. J. Obst. Gyn. $66 ; 475,1953$

24 CASTELAZO AYALA LUIS Y FROGASO LIZALDE DAVID. Curso de Ginecol. y Obst. (Ed. Artes Gráficas). Desprendimiento prematuro de la placenta. Tomo II, pág. 304, 1956.

25 ALVAREZ H. y CALDEYRO-BARCIA R. Fisiopatología de la contracción uterina y sus aplicaciones en la clínica obstétrica. Maternidad e Infancia. 13; 11, 1954.

26 CALDEYRO-BARCIA R., ALVAREZ H., PO. SEIRO J. J., POSE S. V., CIBILS L. A., SI-

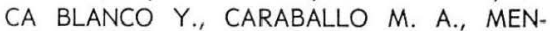
DEZ BAUER C., GONZALEZ PANIZZA V. H. y FIELITZ C. Juicio crítico y resultados de la inducción y conducción del parto. Mem. tercer Congreso Lat. Am. Obst. Gin. Méx. Tomo 1; 131, 1958. 
27 CALDEYRO-BARCIA R. Uterine contractility in Obstetrics. Modern. Trends in Gynecology and Obstetrics. Librairie Beauchemin Limitée. 1: 65, 1958.

28 ALVAREZ H. Y CALDEYRO-BARCIA R. La contractilidad uterina en el desprendimiento prematuro de la placenta. Arch. Gin. Cbst. 9; 1, 1951.

29 COBO EDGAR. La actividad contráctil del útero humano en el abruptio placentae. Rev. Col. Obst. Gin. 14; 221, 1963.

30 FERGUSON J. H. Severe Agruptio placentae. Cln. Obst. and Gyn. 3; 69, 1960.

31 FERGUSON H. J. Hemorrage in late pregnancy. Current Therapy. 656, 1958.

32 HODGKINSON C. P. Premature separation of the normally implanted placentae. Clin. Obst. and Gyn. 3; 585, 1960.

33 HSU CH-T., MA Y-M. et al. Studies on abruptio placentae. Am. J. Obst. Gyn. 80, 263, 1960.

34 KARCHMER K. SAMUEL Y GITLER H. MARIO. Trastornos de la coagulación sanguí- nea en Obstetricia. Rev. Col. Obst. Gin. $19 ; 81,1968$.

35 NEME BUSAMARA. Fundamentos fisiopatológcios del tratamiento del D. P. P. Mem. IV Congreso Mex. Obst. Gin. Tomo II. 154, 1963.

36 GARCIA VALENZUELA RAUL. Criterio terapéutico actual frente a la apoplejía uterina. Mem. IV Congreso Mex. Obst. Gin. Tomo II. 425, 1963.

37 PALACIO SIGIFREDO, CANO ALFONSO $y$ YEPES EDUARDO. Experiencias clínicas con el ácido epsilén amino capróico como agente antifibrinolítico en varios estados hemo. rrágicos. Rev. Col. Obst. Gin. 15; 413, 1964.

38 JAMAIN B., LEROUX M., Mme. BECSLEY et LEROY $M$. La Fibrinolyse en Obstétrique. Eléments actuels du diagnostic biologique. Gyn. et Obst. 64; 621, 1965.

39 STEVENSON CHARLES S. et al. Hemorrhagic diathesis in abruptio placentae: with particular reference to indications for cesaean section. Am. J. Obst. Gyn. 65; 88, 1953. 